\title{
TYPE 2 GAUCHER DISEASE: ONSET AND EVOLUTION - CASE REPORT
}

\author{
Violeta Streanga $^{1}$, Cristina Jitareanu${ }^{1}$, Irina M. Ciomaga ${ }^{1}$, Doina Mihaila ${ }^{2}$, \\ Nicolai Nistor ${ }^{1}$ \\ ${ }^{1} 1^{\text {st }}$ Pediatric Clinic, "Gr. T. Popa" University of Medicine and Pharmacy, Iasi \\ ${ }^{2}$ Department of Pathological Anatomy, \\ "Sf. Maria” Emergency Clinical Hospital for Children, Iasi
}

\begin{abstract}
Gaucher disease is the most common lysosomal storage disorder, with autosomal recessive transmission. The disease is due to glucocerebrosidase enzyme deficiency, resulting in accumulation of glucocerebroside in all organs. The diagnosis is established by measuring enzyme activity. Among the clinical forms, type 2 is the rarest and has the most dismal prognosis. We present the case of an infant found at 5 months of age with neurological symptoms and his subsequent evolution with massive hepatosplenomegaly, being diagnosed with type 2 Gaucher disease.
\end{abstract}

Keywords: Iysosomal storage disorder, type 2 Gaucher disease, neurological manifestations, hepatosplenomegaly, glucocerebrosidase activity

\section{INTRODUCTION}

Gaucher disease is an inherited lisosomal storage disorder characterized by glucocerebroside (glucosylceramide) deposition in cells of the macrophage-monocyte system due to decreased activity of the enzyme beta-glucocerebrosidase (lysosomal enzyme that hydrolyzes glucosylceramide). The disease is recessive autosomal transmitted and is due to a mutation GBA1 gene encoding the enzyme synthesis. In patients with Gaucher disease, over 200 different mutant alleles of the gene have been identified (1).

Glucosylceramide accumulate in the bone marrow, liver, spleen, lung and other organs contributing to pancytopenia, massive hepatosplenomegaly, and, at times, diffuse infiltrative pulmonary disease (1).

The neuronopathic forms of Gaucher disease are characterized by severe neuronal damage, astrocytosis and microglial proliferation. The results of experimental studies carried out on mice with neuronopathic form of Gaucher disease suggest that once the critical threshold of glucosylceramide accumulation is reached in neurons, a cascade of reactions is triggered that activates microglia, which releases inflammatory cytokines that amplify the inflammatory response, contributing to neuronal death (2).

The characteristic Gaucher cells are macrophages lipid loaded with wrinkled paper appearance and displaced nuclei (1).

Depending on the presence or absence of neurologic involvement, three clinical phenotypes have been described:

- Type I - nonneuronopathic form, most frequently encountered;

- Type 2 - acute neuronopathic form, with rapidly progressive neurologic and visceral involvement and death in the first 2 years of life;

- Type 3 - chronic neuronopathic form - the neurovisceral involvements are slowly progressive.

Some patients are affected by severe neurovisceral manifestations in infancy or early childhood

Corresponding author:

Violeta Streanga, "Gr. T. Popa" University of Medicine and Pharmacy, 16 Universitatii Street, lasi

E-mail: streangavioleta@yahoo.com 
but survive beyond 2 years of life, death occurring at the age of 3-7 years; these patients are considered to have an intermediate phenotype between type 2 and 3 disease (1).

For patients with type 1 and 3, enzyme replacement therapy prevents disease progression; for children with type 2 disease treatment is supportive (3).

\section{CASE REPORT}

A 5 months old boy coming from a young, nonconsanguineous couple, with no antenatal or perinatal medical history, was admitted with malaise, apnea, cyanosis and acute respiratory failure due to a bronchopneumonia, whose evolution was favorable after a hospital stay of about two months. The boy had normal weight, increased tone, significant psychomotor delay, convergent strabismus noted by his mother since the age of 3 months and feeding difficulties: swallowing impairment requiring nutrition by tube. TORCH syndrome was suspected, but serology was negative; electroencephalography showed synchronous, diffuse slow activity in all leads. Cerebral CT scan showed mild global cerebral atrophy without any change in density of the cranial contents.

During hospitalization, we noticed hipertonicity, myoclonic episodes in the upper limbs, enlargement of the spleen confirmed by ultrasound: bipolar spleen diameter of $81 \mathrm{~mm}$ (normally: less than $65 \mathrm{~mm}$ for his age and height), increased swallowing difficulties, so consequently we suspected a lisosomal storage disorder. A bone marrow aspirate did not contain any specific pathological cells; the marrow was effective and not infiltrated. A mild microcytic hypochromic anemia was noted and also normal liver function. The diagnosis was cerebral palsy, global cerebral atrophy, anemia, and the recommended treatment was Phenobarbital and iron preparations. The infant was discharged at the request of his family and admitted in an orphanage, as he needed nutrition by tube.

Afterwards, the child was hospitalized twice, at the age of 8 and, respectively, 9 months, for acute respiratory diseases. We observed: mild failure to thrive, axial hypertonicity, hyperextension of the neck with opisthotonus, laryngeal stridor, convergent strabismus, swallowing impairment requiring feeding by tube, severe neuromotor delay, hepatosplenomegaly, persisted mild hypochromic microcytic anemia. Chest X-ray revealed interstitial infiltration issue. The association of hepatosplenomegaly with severe psychomotor retardation and swallow- ing disorders led us to reconsider a lysosomal storage disease. Testing performed at the age of 9 months revealed low activity for beta-glucocerebrosidase: $98,04 \mathrm{pmol} / \mathrm{spot} 20 \mathrm{~h}$, representing $8,9 \%$ of the average normal activity, raising suspicion of type 2 Gaucher disease. Sphingomyelinase activity, alpha-iduronidase and beta-galactosidase activity were scored in the normal range, discarding the suspicion of Niemann-Pick disease, and mucopolysaccharidosis. Treatment was supportive.

At the age of 10 months and 3 weeks the infant was admitted after a prolonged apnea episode; clinically, we noticed: massive hepatosplenomegaly, failure to thrive, hyperextension of the neck with opisthotonus, progressive psychomotor regression, maintaining swallowing disorders, tonic and tonicclonic seizures, laryngeal stridor and frequent and prolonged apnea episodes. Another measurement of beta-glucocerebrosidase activity in patient's leukocyte was $35,27 \mathrm{pmol} / \mathrm{spot} 20 \mathrm{~h}$, representing $3,2 \%$ of the average normal activity, confirming the diagnosis of type 2 Gaucher disease. Death occurred at the age of 12 months by Pseudomonas Aeruginosa bronchopneumonia. Parents refused necropsy, allowing us only to take a sample of the liver and the intestine through a small incision in the right flank. Pathological examination revealed: liver architecture destroyed by the presence of a histiocytic infiltrate dispersed among the hepatocytes; this histiocytes had abundant, pale eosinophilic cytoplasm, looking fibrilar or homogeneous, also central or peripheral core (Fig. 1); intestine histiocytic infiltrate with eosinophilic cytoplasm in the area of lymphoid follicles (Fig. 2).

Two years later, the parents had the molecular test done: the father was heterozygous for L444P mutation - one of the most common mutation ob-

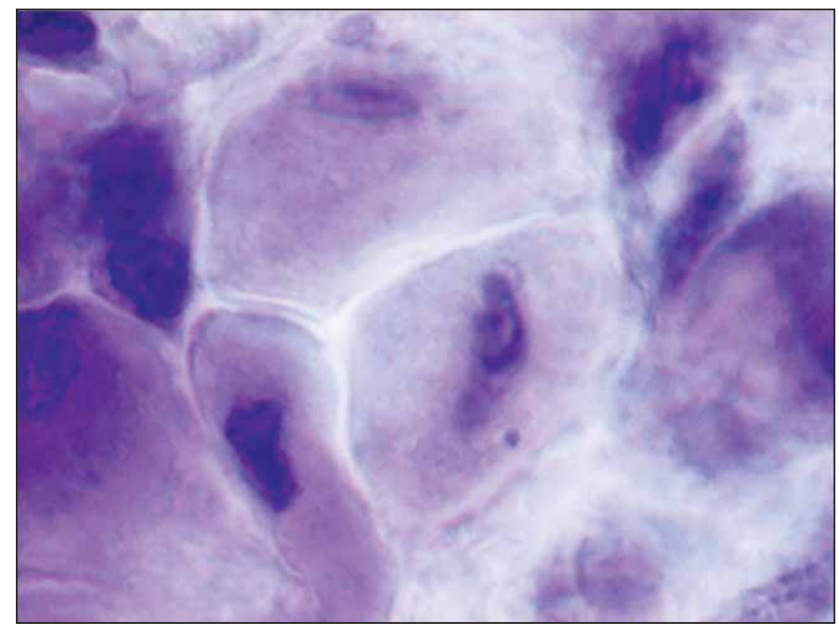

FIGURE 1. Gaucher cells inside the liver. Section in frozen tissue and Scharlach stain $\times 1000$ 


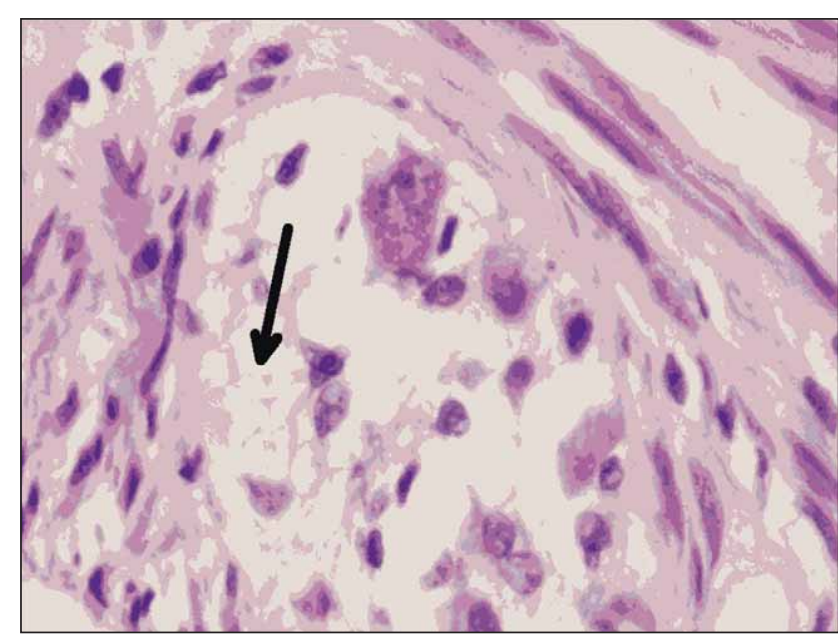

FIGURE 2. Gaucher cells inside the myenteric plexus of the small intestine. HE $\times 400$

served in the non-Jewish population, and the mother was heterozygous for a rare mutation: T134I.

\section{DISCUSSION}

Type 2 Gaucher disease begins in the first 3-6 months of life, leading to progressive neuronal degeneration and death in the first 2 years of life; the incidence of this form of the disease is rare, estimated at $1 / 150000$ (4).

Two clinical subtypes have been described:

- Acute neuronopathic form, with clinical onset after a symptoms free interval of 3-6 months of life and death before the age of 2 years;

- Neonatal-lethal form characterized by nonimun fetal hydrops, congenital ichthyosis, facial dysmorphism with perinatal or early mortality in uterus.

The clinical manifestations of the classic form of Gaucher type 2 include hypertonia, seizures, strabismus, organomegaly, swallowing disorders, failure to thrive, stridor by laryngospasm, progressive psychomotor retardation; death can occur by aspiration or respiratory impairment (1). The most frequent initial signs are hyperextension of the neck, swallowing disorders and strabismus (5), but there were cited cases of neonatal cholestasis as early onset of the disease (4).

Differential diagnosis is necessary with Niemann Pick disease, easily accomplished by determining the enzymatic activity.

Diagnosis can be confirmed by measuring of glucocerebrosidase activity in peripheral blood leukocytes. A value below $15 \%$ of mean normal activ- ity is diagnostic. Heterozigotes generally have halfnormal enzyme activity (1).

Molecular testing can identify mutations. Further investigations are needed: blood count to assess cytopenia; transaminases - can register a minor elevation; ultrasound for organomegaly assessment; chest-X ray to assess lung function. Bone marrow aspiration or liver biopsy can identify Gaucher cell, but the enzyme assay is sensitive, specific, and much less invasive (1).

Treatment is supportive for this form of disease, because enzyme replacement therapy cannot reverse the neurological manifestations, due to recombinant enzyme's inability to cross the bloodbrain barrier. Thus, the current enzyme replacement therapy is limited to the treatment of non-neurological symptoms (6).

Substrate reduction therapy and pharmacological chaperone therapy are other ongoing research strategies for these patients.

For the families which include a member diagnosed with type 2 or 3 Gaucher disease, it is mandatory to follow genetic counseling, which should contain direct enzymatic assay of beta-glucocerebrosidase and molecular testing of the GBA mutations on chorionic villous samples (7).

The case we presented is a type 2 Gaucher disease with onset in the first 6 months of life; the initial symptoms were psychomotor retardation, impaired swallowing and strabismus. Because we didn't find Gaucher cells in the bone marrow aspirate, the diagnosis was delayed. Subsequently, clinical signs included organomegaly, becoming gradually massive, hyperextension of the neck with opisthotonus and failure to thrive, thus correct diagnosis was established at the age of nine months, by measuring enzymatic activity.

\section{CONCLUSION}

We concluded that neurological manifestations detected in the first months of life may be the first clinical signs of a lysosomal storage disorder. The lack of Gaucher cells in bone marrow aspirate does not exclude the diagnosis of type 2 Gaucher disease, yet the positive diagnosis is confirmed by measuring glucocerebrosidase enzyme activity in peripheral blood leukocytes.

\section{Acknowledgements}

We would like to thank Dr. Z. Lukacs (Metabolic Laboratory, Hamburg University Medical Center, Hamburg, Germany) for his invaluable help in measuring the activity of beta-glucocerebrosi- 
dase. We also thank the Genzyme, a Sanofi Company, for its constant support. The authors are also grateful to the infant's parents, who accepted the report of the case and provided us the result of their genetic testing.

\section{REFERENCES}

1. Sidransky E., Steiner R.D., Windle M.L. et al. Gaucher Disease. http://emedicine.medscape.com/article/944157. Accesat 7 Mai 2014.

2. Vitner E.B., Farfel-Becker T., Eilam R. et al. Contribution of brain inflammation to neuronal cell death in neuronopathic forms of Gaucher's disease. Brain 2012; 135(Pt 6): 1724-35.

3. Kaplan P., Baris H., De Meirleir L. et al. Revised recommendations for the management of Gaucher disease in children. Eur J Pediatr 2013; 172(4): 447-58.

4. Elias A.F., Johnson M.R., Boitnott J.K., Valle D. Neonatal cholestasis as initial manifestation of type 2 Gaucher disease: a continuum in the spectrum of early onset Gaucher disease. JIMD Rep. 2012; 5: 95-8.

5. Mignot C., Doummar D., Maire I., De Villemeur T.B. French Type 2 Gaucher Disease Study Group. Type 2 Gaucher disease: 15 new cases and review of the literature. Brain Dev 2006; 28(1): 39-48.

6. Grabowski G.A. Phenotype, diagnosis, and treatment of Gaucher's disease. Lancet. 2008; 372: 1263-1271.

7. Germain D.P., Benistan K. Prenatal diagnosis of Gaucher disease. Rev Med Interne 2007; 28 Suppl 2: S193-7. 